\title{
The Radiation System Synthesis by the Power Criterion as a Problem of Optimization with Restrictions
}

\author{
Mykhaylo I. Andriychuk, Bohdan M. Podlevskyi \\ Department of Numerical Methods of Mathematical Physics, Pidstryhach Institute for Applied Problems of Mechanics \\ and Mathematics, NASU, Lviv, Ukraine \\ Email: andr@iapmm.lviv.ua,bpodlev@gmail.com
}

How to cite this paper: Andriychuk, M.I. and Podlevskyi, B.M. (2018) The Radiation System Synthesis by the Power Criterion as a Problem of Optimization with Restrictions. Journal of Applied Mathematics and Physics, 6, 2650-2665.

https://doi.org/10.4236/jamp.2018.612220

Received: December 1, 2018

Accepted: December 24, 2018

Published: December 27, 2018

Copyright (c) 2018 by authors and Scientific Research Publishing Inc. This work is licensed under the Creative Commons Attribution International License (CC BY 4.0).

http://creativecommons.org/licenses/by/4.0/

\begin{abstract}
A nonlinear synthesis problem of antennas according to the prescribed power (squared amplitude) radiation pattern (RP) is considered in the variational statement that yields in the possibility to take into account an additional restriction to the synthesized power RP. The problem of synthesis consists of finding such currents in antenna, which generates the RP with the best approximation to the given one. The respective Euler's equation is reduced on the basis of used functional. This is nonlinear integral equation of Hammerstein's type. The effective numerical methods are elaborated and applied for its solving. The computational results verify the effectiveness of approach proposed.
\end{abstract}

\section{Keywords}

Power RP, Variational Approach, Non-Linear Integral Equation, Successive Approximation Method

\section{Introduction}

In the process of design the various radiating systems, in particular antennas and arrays, requirements are imposed mainly on the amplitude or power RP; the phase characteristic (the argument of complex RP) remains free of any requirements. The antenna synthesis problems according to amplitude characteristics are extensively investigating in the recent decade for plane arrays with different geometries [1] [2] [3], for the case of specific form of the given RP [4], as wells as due to moving the antenna techniques to new perspective $\mathrm{THz}$ range of frequencies [5]. 
In the problems of optimization, as well as in related to this area the synthesis problems according to the amplitude or power RP [6], the phase RP can be used as an additional optimization parameter, which allows obtain the better approximation to the prescribed RPs.

The first works in this area were [7] [8], in which the synthesis problem of a linear antenna by a given amplitude RP was formulated as a variational problem of minimizing the mean square deviation of the given (real positive function) and amplitude of the synthesized RPs. The obtained Euler's equation for a minimizing functional was a nonlinear integral one. Since such equation describes not only the extreme points of the considered functional, but also all its possible stationary points, its solution may be ambiguous. Moreover, the number of solutions can vary with the change of physical parameters of problem (process of branching the solutions). This property was described and discussed firstly in [9]. The approach proposed was applied for different types of antennas [10] and generalized in [11]. The theoretical investigations related to study of branching process for one- and two-dimensional cases were conducted in [12] [13] [14] [15] [16].

The formulation of the synthesis problem of antennas according to the power RP was apparently proposed for the first time in [17] as a modification of the synthesis problem by amplitude RP. In the later works [18] [19] [20] [21] such statement of problem was described and applied for one-dimensional and two-dimensional antennas. Recently such a statement was supplemented with the condition of the norms' equality of synthesized and given power RPs [22]. The corresponding nonlinear integral equation was obtained; it was solved by the modified Newton's method. Since this equation has a non-unique solution, not all of these solutions have been obtained.

The above disadvantage can be overcome using a polynomial approach to synthesis problems according to the amplitude RP [23] [24]. This approach is based on the exact representation of solutions to the respective nonlinear integral equation by complex polynomials of low degree; it was generalized and described in detail in [6]. Its application to the problem of antenna synthesis by the power criterion is given in [25] [26] [27].

In this paper, using the variational statement of synthesis problem, we elaborate the numerical methods for the linear antenna and equidistant array. The additional restriction on the norm of synthesized power RP is taken into account in the presented functional, that is, the synthesis problem is formulated as a problem of conditional minimization of used functional with constraints. The formulation of problem uses a general operator representation of the synthesized RPs by the currents in antenna. Study of properties of solutions to the respective Euler's equations for linear antenna is focused more on the theoretical investigation related to branching process, because the properties of direct and adjoint operators are simpler in this case that allow deal with some analytical transformations. Since the above operators for plane array are more complicate, then for this antenna we deal mainly with synthesis of the specific prescribed power RPs. 


\section{Formulation of the Problem}

It is known that the RP of the radiation system is a vector complex-valued function and its dependence on the distribution of currents in system has a linear character. Abstracting from the specific type of the radiation system, this dependence can be written with the help of some linear operator $A$

$$
f=A u,
$$

where $f$ is RP created (synthesized) by radiation system, $u$ is current distribution in system.

The form and properties of the operator $A$ are determined by the type and geometry of the radiating system.

The synthesis problem according to the power RP is formulated as a problem of minimizing the functional

$$
\sigma(u)=\left\|F^{2}-|f|^{2}\right\|_{2}^{2}+\alpha\|u\|_{1}^{2} .
$$

Here $\|\cdot\|_{1},\|\cdot\|_{2}$ are the mean-square norms in the spaces of functions $u$ and $f$, respectively; $F^{2} \geq 0$ is the given power RP within a certain area $\Omega$, and $\alpha>0$ is the given real parameter. The first term in (2) ensures the proximity of the synthesized power RP to a given one in the main lobe (region $\Omega$ ), while the second term imposes restrictions on the norm of exciting currents. We obtain the equations for the synthesized power RP

$$
f=\frac{2}{\alpha} A A^{*}\left[\left(F^{2}-|f|^{2}\right) f\right]
$$

from the necessary condition of functional (2) minimum, here $A^{*}$ is operator adjoint to $A$.

Note that Equation (3) has a obvious trivial solution $f \equiv 0$ always. To avoid this undesirable property, as well as to limit the trend of proportional reduction of the RP $f$ with increasing in the $\alpha$ coefficient, the functional to be supplemented by the additional condition

$$
\|f\|_{2}^{2}=\|F\|_{2}^{2} .
$$

This means that we will consider the problem of minimizing the functional with constraints on the RP in the region $\Omega$.

The application of the Lagrange multiplier method to the optimization problem (2), (4) leads to a minimization of functional

$$
L(u, \lambda)=\sigma(u)+\lambda\left(\|f\|^{2}-\|F\|^{2}\right)
$$

where $\lambda$ is the Lagrange multiplier, which along with $f$ is also be determined from the minimum of the functional (5).

The Euler equation of functional (5) can be written in form

$$
\alpha f-2 A A^{*}\left[\left(F^{2}-|f|^{2}\right) f\right]-\lambda A A^{*} f=0 \text {. }
$$

If $f$ and $\lambda$ are found from (6), then the optimal current $u$ is calculated by 
formula

$$
u=\frac{1}{\alpha}\left(2 A^{*}\left[\left(F^{2}-|f|^{2}\right) f\right]+\lambda A^{*} f\right) .
$$

Equation (6) can be supplemented by condition (4), then they are considered as a system of equations for determining $f$ and $\lambda$.

\section{Methods of Solution}

\subsection{Usual Iterative Method}

We propose different methods for solving the problem of antenna synthesis, which are reduced to the search for a stationary point of functional (5). For example, it is naturally to apply the iterative process of the gradient method for both the unknowns $u$ and $\lambda$ :

$$
\begin{gathered}
u^{(k+1)}=u^{(k)}-\gamma_{k} L_{u}\left(u^{(k)}, \lambda_{k}\right), \\
\lambda_{k+1}=\lambda_{k}+\gamma_{k} L_{\lambda}(u, \lambda)=\lambda_{k}+\gamma_{k}\left(\|f\|^{2}-\|F\|^{2}\right),
\end{gathered}
$$

$k=0,1, \cdots$, where $L_{u}(u, \lambda)=\left(L_{u_{1}}(u, \lambda), \cdots, L_{u_{n}}(u, \lambda)\right), \quad L_{\lambda}(u, \lambda)=\|f\|^{2}-\|F\|^{2}$, while the value of the step $\gamma_{k}$ in (8), (9) can be chosen for the same reasons as for unconditional optimization.

Instead of (8) one can use other iterative processes, in particular the Newton's method, which is described in the next subsection. In our case, the problem of minimizing function $L(u, \lambda)$ by variable $u$ is solved quite simply, so we offer the following iteration process:

$$
\begin{gathered}
\min _{u \in U_{0}} L\left(u_{k}, \lambda_{k}\right), \\
\lambda_{k+1}=\lambda_{k}+\gamma_{k}\left(\|f\|^{2}-\|F\|^{2}\right), k=0,1, \cdots
\end{gathered}
$$

\subsection{Modified Newton's Method}

In general, Equation (6) can be written in form

$$
\Phi(f, c)=0,
$$

where $\Phi$ is nonlinear operator-function, $f$ is synthesized RP, $c$ is numerical parameter characterizing the physical performances of antenna (as a rule it is value that combines the size of antenna and frequency of excitation).

If for a certain $c$ there is a function $f$ satisfying Equation (12), then we call the pair $(f, c)$ a solution of this equation. If $f$ continuously depends on $c$, then we assume that the solution of Equation (12) belongs to a certain branch of the solutions, or is a "point" on this branch. Below, we propose such a modification of the Newton's method, which will allow to find such branches, as well as points of branching on them, if they exist.

Let the pair $\left(f_{n}, c_{n}\right)$ be $n$-th approximation to the desired point on the branch of the solutions. The next $n+1$-th approximation is found from the condition that the Equation (12) is satisfied in the first order by increments 
$\varepsilon=c_{n+1}-c_{n}$ and $\delta=f_{n+1}-f_{n}$. This requirement yields in the following equation

$$
\Phi_{c} \cdot \varepsilon+\Phi_{f}\left(f_{n}, c_{n}, \delta\right)=-\Phi_{n}
$$

where

$$
\Phi_{c}=\left.\frac{\partial \Phi}{\partial c}\right|_{c=c_{n}},
$$

and $\Phi_{f}\left(f_{0}, c_{0}, \delta\right)$ is determined from the condition

$$
\Phi(f, c)=\Phi_{n}+\Phi_{c} \cdot \varepsilon+\Phi_{f}\left(f_{n}, c_{n}, \delta\right)+o(\varepsilon, \delta),
$$

and $\Phi_{n}=\Phi_{f}\left(f_{n}, c_{n}\right)$. Equation (13) is linear with respect to $\varepsilon$ and $\delta$. If it is solved, the next approximation of solution is determined by formula

$$
c_{n+1}=c_{n}+\varepsilon, f_{n+1}=f_{n}+\delta .
$$

Obviously, the Equation (12) is undetermined. If the iterative process converges to some values, then its limit, generally speaking, is an arbitrary point on the branch. The Equation (13) is also undetermined and it can have a certain set of solutions. If no additional conditions are imposed on the solution $f$, it is possible, in particular, to choose a solution with a minimum norm among the set of solutions, that is, the "point" of this set closest to the previous approximation will be selected for the next approximation. This choice avoids a situation where a new point on a branch is either too far or very close to the last point that has already been found. One can also require that the distance between the points on a branch during computations be kept constant by prescribing this distance, for example, by means of equality

$$
|\Delta c|^{2}+\|f\|_{2}^{2}=h^{2}
$$

where $\Delta c=c_{n-1}-c_{n}, \Delta f=f_{n}-f_{n-1}$, and indices $n-1$ and $n$ meaning affiliation of the value to the previous step and to the desired point on the branch in the current step. The above equality complements the Equation (12). In this case, the Equation (13) must be supplemented by the equation

$$
\Delta c_{n} \cdot \varepsilon+\left(\Delta f_{n}, \delta\right)=0,
$$

which follows from the perturbation (16). As a result, the system of Equation (13), (18) becomes defined. In general, this system is equivalent to initial system of Equation (4), Equation (6).

Without decreasing universality, the condition (4) for the effective use of the generalized Newton's method can be written in the form.

$$
\|f\|_{2}^{2}=1 \text {. }
$$

In order to solve this system we use the modified Newton's method proposed in [6]. For this goal, we rewrite Equation (6), Equation (19) in form

$$
\begin{gathered}
\Phi(f, \mu) \equiv \alpha f-2 A A^{*}\left[\left(F^{2}-|f|^{2}\right) f\right]-\lambda A A^{*} f=0, \\
\Psi(f) \equiv\|f\|_{2}^{2}-1=0 .
\end{gathered}
$$


The next approximation to unknowns $f$ and $\lambda$ in this method is calculated as

$$
\begin{gathered}
f_{n+1}=f_{n}+\delta f_{n}^{\prime}+i \delta f_{n}^{\prime \prime}, \\
\lambda_{n+1}=\lambda_{n}+\delta \lambda_{n},
\end{gathered}
$$

where values $\delta f_{n}^{\prime}, \delta f_{n}^{\prime \prime}, \delta \lambda_{n}$ are determined as solutions of nonlinear system of equations

$$
\left[\begin{array}{ccc}
\alpha-\lambda_{n} A A^{*}-2 A A^{*}\left(F^{2}\right. & 4 A A^{*}\left(f_{n}^{\prime} f_{n}^{\prime \prime}\right) & -A A^{*}\left(f_{n}^{\prime}\right) \\
\left.-\left|f_{n}\right|^{2}\right)+4 A A^{*}\left(f_{n}^{\prime}\right) & & \\
4 A A^{*}\left(f_{n}^{\prime} f_{n}^{\prime \prime}\right) & \alpha-\lambda_{n} A A^{*}-2 A A^{*}\left(F^{2}\right. & -A A^{*}\left(f_{n}^{\prime \prime}\right) \\
2 f_{n}^{\prime} & \left.-\left|f_{n}\right|^{2}\right)+4 A A^{*}\left(f_{n}^{\prime \prime}\right) & 0
\end{array}\right]\left[\begin{array}{l}
\delta f_{n}^{\prime} \\
\delta f_{n}^{\prime \prime} \\
\delta \lambda_{n}
\end{array}\right]=\left[\begin{array}{l}
-\Phi_{n}^{\prime} \\
-\Phi_{n}^{\prime \prime} \\
-\Psi_{n}
\end{array}\right]
$$

In the case if parameter $\lambda$ is fixed then Equation (20) is not in use in the system, therefore the last row and column in (24) are non-available.

\section{Application to Specific Antennas and Arrays}

The iterative procedures, elaborated in the previous Section are applied here for solving the synthesis problems for the specific antennas. Both the linear antenna and plane equidistant array are considered. The modified Newton's method is applied for the synthesis of linear antenna. This is because of fact that the operators $A$ and $A^{*}$ for the plane array need more calculations that the respective operators for linear antenna. Because the usual iterative procedure (8), (9) is simpler, the calculations can be applied for more complicate antennas.

\subsection{Linear Antenna}

The proposed approach is applied in this subsection to synthesis of linear antenna. In this case, operators $A, A^{*}$, and kernel $K\left(\xi_{1}, \xi_{2}\right)$ of operator $A A^{*}$ has form

$$
\begin{gathered}
f(\xi)=A u \equiv \int_{-1}^{1} u(x) \mathrm{e}^{i c x \xi} \mathrm{d} x, \\
A^{*} g=\frac{c}{2 \pi} \int_{-1}^{1} g(\xi) \mathrm{e}^{-i c x \xi} \mathrm{d} \xi \\
K\left(\xi_{1}, \xi_{2}\right)=\frac{\sin c\left(\xi_{1}-\xi_{2}\right)}{\pi\left(\xi_{1}-\xi_{2}\right)}
\end{gathered}
$$

where $x$ is normalized coordinate in antenna, $\xi=\sin \varphi / \sin \varphi_{0}$ is generalized angular coordinate in far zone, $2 \varphi_{0}$ is angle within the given power RP differs on zero, $c=k a \sin \varphi_{0}, k$ is wavenumber, $2 a$ is length of antenna.

The Euler equation for functional (5) has form

$$
\begin{aligned}
& \alpha f\left(\xi^{\prime}\right)-\frac{2}{\pi} \int_{-1}^{1} \frac{\sin c\left(\xi-\xi^{\prime}\right)}{\pi\left(\xi-\xi^{\prime}\right)}\left[\left(F^{2}(\xi)-|f(\xi)|^{2}\right) f(\xi)\right] \mathrm{d} \xi \\
& -\lambda \int_{-1}^{1} \frac{\sin c\left(\xi-\xi^{\prime}\right)}{\pi\left(\xi-\xi^{\prime}\right)} f(\xi) \mathrm{d} \xi=0
\end{aligned}
$$


The numerical results are presented for the given power RPs $F^{2}(\xi)=0.5$ and $F^{2}(\xi)=\cos (\pi \xi / 2),|\xi|<1$; these functions are equal to zero if $|\xi|>1$. The value of constant 0.5 for the first given RP is chosen from the condition that its norm within the interval $[-1,1]$ be equal to 1 . This yields simplification in the computational scheme of method.

The main interest from the engineering point of view presents the mean-square deviation $\sigma_{0}=\left\|F^{2}-|f|^{2}\right\|_{2}^{2}$ of power RPs (first term in functional (5)), the rest of terms have the auxiliary importance. In Figure 1, the dependence of $\sigma_{0}$ value on parameter $c$ is shown for the different solutions to Equation (28). One can see that the quality of approximation depends essentially on $\alpha$ parameter (weight multiplier in functional (2)). The branching of solutions appears at the specific values of $c$, these values are marked by $c_{n m}$; index $n$ corresponds to $\alpha$ value ( $n=1$ corresponds to $\alpha=0.1 ; n=2$ corresponds to $\alpha=0.4 ; \alpha=0.4$ corresponds to $\alpha=0.9$ ), and index $m$ corresponds to number of branching point.

For two given RPs $F^{2}(\xi)$ at all values of $c$ (dashed lines in the figures), there are real solutions. The difference in behaviour of $\sigma_{0}$ for various $F^{2}(\xi)$ at small $c$ is due to the fact that the real solution of Equation (28) is asymptotically constant at $c \rightarrow 0$. Therefore, function $F^{2}(\xi) \equiv$ const may be better approximated for small $c$. This property is valid only for this given power RP $F^{2}(\xi)$.

One should note that at $\lambda=0$ and fixed $\alpha$ Equation (28) has only a zero solution for small $c$. This is due to the fact that the second term in the functional (5) is dominant for small $c$.

A solution with an odd phase $\arg f(-\xi)=-\arg f(\xi)$ (continuous curves) branches off at points $c=c_{n 1}$ from a real solution. Branching points coincide approximately with the points of the first maximum of $\sigma_{0}$ as a function of the parameter $c$ for a real solution. It follows from equation (28) that the current distribution $u(x)$ in the antenna, which forms a RP with an odd phase $\arg f(\xi)$ is real, but it crosses through zero point on the interval $x \in[-1,1]$. This fact is significant from an engineering point of view, because there is no need to use a phase corrector for its implementation.

The point $c_{n 2}$ is the next characteristic point in Figure 1, where two new complex solutions arise simultaneously with the odd and even phase. They have the same $|f(\xi)|$ and respectively the same $\sigma_{0}(u)$. But the current $u(x)$ is different for these two solutions. One that corresponds to an odd $\arg f(\xi)$, is real and it crosses through zero point in the interval $x \in[-1,1]$, while the second one, with the even phase $(\arg f(-\xi)=\arg f(\xi)$ ), is an even complex functions (in some cases, it can also cross through zero point). Solutions with the odd phase are branching off the solutions of the same type (that is, they branch off the solution having an odd phase), while the solutions with even phase are branched off the real solution; both of these types of solutions appear at the same point $c_{n 2}$. Thus, there are at least four solutions: real (that is, with a zero phase), one with an even phase, and two with an odd phase at values $c>c_{n 2}$. In Figure 1 , the results are presented for one solution with odd phase only. 


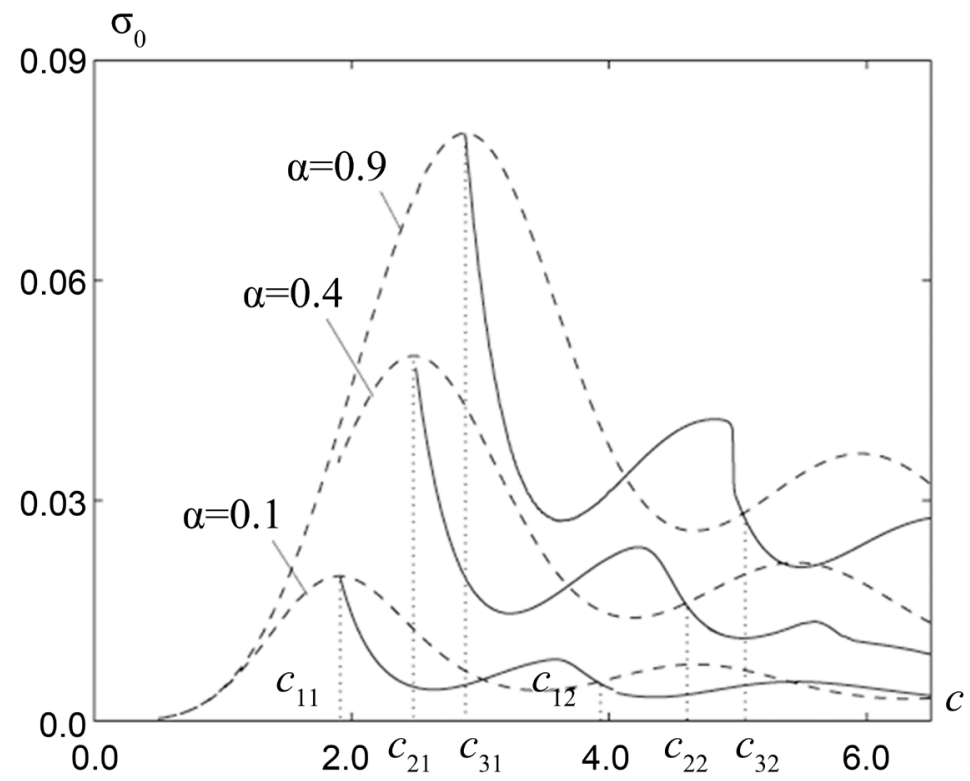

(a)

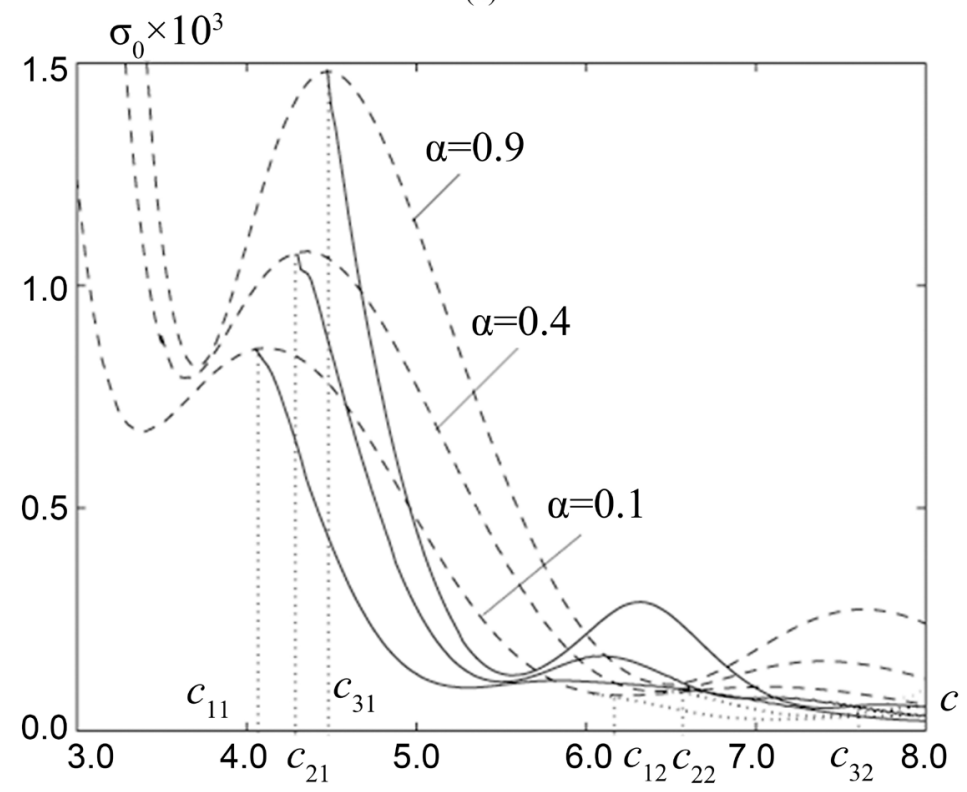

(b)

Figure 1. Mean-square deviation of power RPs for different solutions to equation (28): (a) $F^{2}(\xi)=1 / 2 ;$ (b) $F^{2}(\xi)=\cos (\pi \xi / 2)$.

Note that the parity of phase distributions $\arg f(\xi)$ and $\arg u(x)$ is related to the symmetry of a given $\mathrm{RP} F^{2}(\xi)$ and both intervals $x \in[-1,1]$ and $\xi \in[-1,1]$.

The presented numerical results show that the properties of solutions of the synthesis problem by the power RP are qualitatively close to the properties of the solutions of the synthesis problem by a given amplitude RP (in [28] [29] exact solutions of the last problem are obtained). Nevertheless, this analogy can not be complete, since the problem under consideration here has higher order nonlin- 
earity and may have additional solutions that differ from the properties of the above-mentioned problem.

At the fixed $c$, the current norm is almost independent on $\alpha$ for the solutions of all types. This is explained by the fact that this norm is essentially determined by the norm of the synthesized RP, which is fixed in our statement.

In Figure 2, the qualitative characteristics of the synthesis results are presented for fixed value $c=5.0$ at $\alpha=0.4$ for prescribed constant power RP $F^{2}(\xi)=0.5$. The numbers in Figure 2 correspond to the following types of phase of the synthesized power RP: 1 corresponds to real synthesized RP $|f|^{2}$ with zero (constant) phase; 2 corresponds to synthesized RP $|f|^{2}$ with even

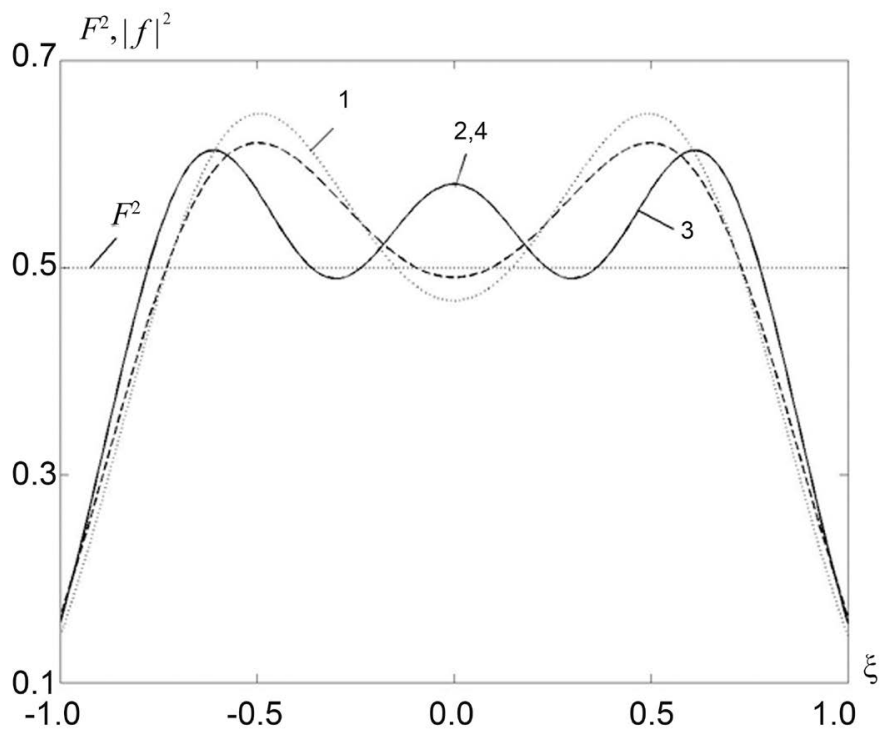

(a)

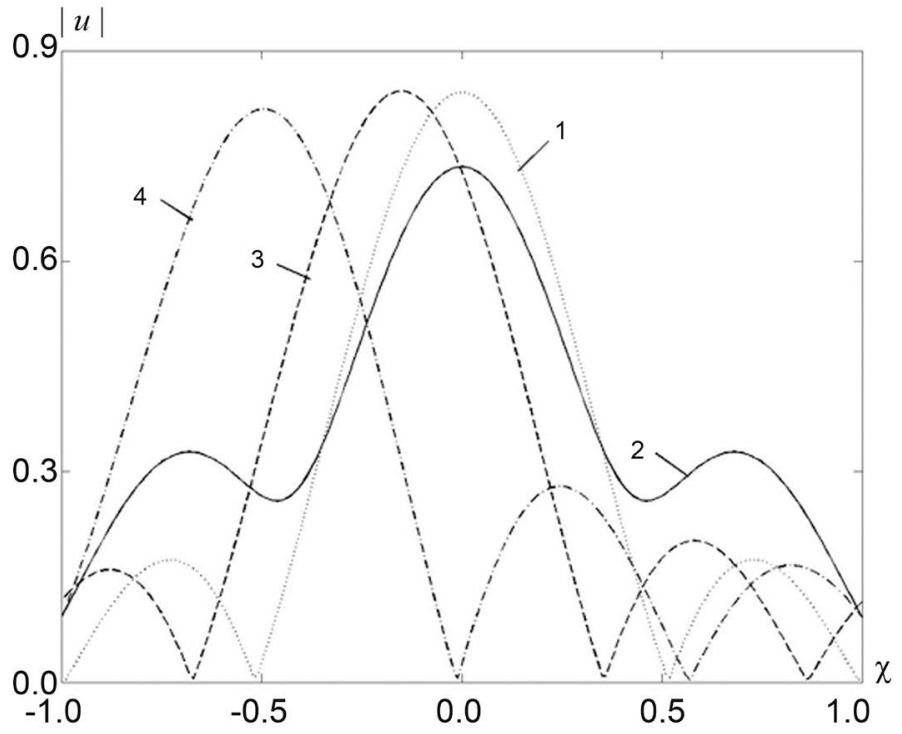

(b)

Figure 2. The synthesized power RPs (a) and respective amplitude distributions of currents (b) for $F^{2}(\xi)=0.5, c=5.0, \alpha=0.4$. 
phase; 3 corresponds to first synthesized RP $|f|^{2}$ with odd phase; 4 corresponds to second synthesized RP $|f|^{2}$ with odd phase. As follows, there are four solutions to Equation (28) that differ by the various types of phase of the synthesized power RP. The specific properties of such kind of solutions is that the amplitude of synthesized power RP $|f|^{2}$ is symmetrical for all types of solutions, despite the fact that the amplitude $|u|$ of optimal currents are non-symmetrical for solutions 3 and 4 . The above properties are identical those were obtained for solution of synthesis problem without taking into account the restriction on the norm of the synthesized RP [6].

\subsection{Plane Array}

Consider a plane array consisting of $N=\left(2 M_{1}+1\right) \times\left(2 M_{2}+1\right)$ of identical and identically oriented radiators with the same RP of all radiators, in which the phase centre is located on the plane $\mathrm{xOy}$ (plane of array) of the Cartesian coordinate system. We assume that the coordinates of the centres of radiators $\left(x_{n}, y_{m}\right)$ form a rectangular equidistant lattice, oriented along the axis and symmetric with respect to these axes. Then the function describing the RP (array multiplier) has the form

$$
f(\theta, \varphi)=\sum_{n=-M_{1}}^{M_{1}} \sum_{m=-M_{2}}^{M_{2}} I_{n m} \mathrm{e}^{i k\left(x_{n} \sin \theta \cos \varphi+y_{m} \sin \theta \sin \varphi\right)},
$$

where $I_{n m}$ is the distribution of the complex currents in $n m$-th radiator, $\theta, \varphi$ are the angular coordinates of the spherical coordinate system $(R, \theta, \varphi)$ whose centre coincides with the centre of the Cartesian coordinate system $x O y$. It is assumed in (29) that the RPs of separate radiators do not depend on the coordinates $\theta$ and $\varphi$, that is they are constant (omnidirectional).

We introduce generalized variables $\tilde{\xi}_{1}=\sin \theta \cos \varphi, \quad \tilde{\xi}_{2}=\sin \theta \sin \varphi$ and denote by $d_{1}$ and $d_{2}$ distances between neighbouring radiators along the $O x$ and $O y$ axes respectively. Then the coordinates of the radiators are calculated using relation $x_{n}=d_{1} n, y_{m}=d_{2} m$, and the array multiplier (29) can be presented in the form

$$
f\left(\tilde{\xi}_{1}, \tilde{\xi}_{2}\right)=\sum_{n=-M_{1}}^{M_{1}} \sum_{m=-M_{2}}^{M_{2}} I_{n m} \mathrm{e}^{i\left(\tilde{c}_{1} n \tilde{\xi}_{1}+\tilde{c}_{2} m \tilde{\xi}_{2}\right)},
$$

where $\tilde{c}_{1}=k d_{1}, \tilde{c}_{2}=k d_{2}$.

Note that function $f\left(\tilde{\xi}_{1}, \tilde{\xi}_{2}\right)$ is a periodic one with the period $2 \pi / \tilde{c}_{1}$ for the variable $\tilde{\xi}_{1}$ and with the period $2 \pi / \tilde{c}_{2}$ for the variable $\tilde{\xi}_{2}$. Let us denote by $R_{2}$ the region that corresponds to one period $R_{2}:\left\{\left|\tilde{\xi}_{1}\right| \leq \pi / \tilde{c}_{1},\left|\tilde{\xi}_{2}\right| \leq \pi / \tilde{c}_{2}\right\}$ and assume that the required power $\operatorname{RP} F^{2}\left(\tilde{\xi}_{1}, \tilde{\xi}_{2}\right)$ is given in some area $\Omega \subset R_{2}$, for example, $\Omega:\left\{\left|\tilde{\xi}_{1}\right| \leq b_{1},\left|\tilde{\xi}_{2}\right| \leq b_{2}\right\}$ and it is described by a function that is continuous and non-negative in the domain $\Omega$ and identically equal to zero outside $\Omega$. Let us denote by $2 \alpha_{1}$ and $2 \alpha_{2}$ the intervals of the change of angle $\theta$ in the region $\Omega$, for $\varphi=0$ and $\varphi=\pi / 2$, respectively, and introduce new variables $\xi_{1}=\tilde{\xi}_{1} / \sin \alpha_{1}, \quad \xi_{2}=\tilde{\xi}_{2} / \sin \alpha_{2}$. Then $\Omega:\left\{\left|\xi_{1}\right| \leq 1,\left|\xi_{2}\right| \leq 1\right\}$, and the array multiplier can be written as 


$$
f\left(\xi_{1}, \xi_{2}\right)=\sum_{n=-M_{1}}^{M_{1}} \sum_{m=-M_{2}}^{M_{2}} I_{n m} \mathrm{e}^{i\left(c_{1} n \xi_{1}+c_{2} m \xi_{2}\right)},
$$

where $c_{1}=k d_{1} \sin \alpha_{1}, c_{2}=k d_{2} \sin \alpha_{2}$.

Functional (5) for this case has form

$$
\begin{aligned}
L(I, \lambda)= & \int_{-1}^{1} \int_{-1}^{1}\left[F^{2}\left(\xi_{1}, \xi_{2}\right)-\left|f\left(\xi_{1}, \xi_{2}\right)\right|^{2}\right]^{2} \mathrm{~d} \xi_{1} \mathrm{~d} \xi_{2}+\sum_{n=-M_{1}}^{M_{1}} \sum_{m=-M_{2}}^{M_{2}}\left|I_{n m}\right|^{2} \\
& +\lambda \int_{-1}^{1} \int_{-1}^{1}\left[F^{2}\left(\xi_{1}, \xi_{2}\right)-\left|f\left(\xi_{1}, \xi_{2}\right)\right|^{2}\right] \mathrm{d} \xi_{1} \mathrm{~d} \xi_{2}
\end{aligned}
$$

We obtain a nonlinear system of equations for finding the current distribution on radiators

$$
\begin{gathered}
\alpha I_{n m}=\int_{-1}^{1} \int_{-1}^{1}\left(2\left[F^{2}\left(\xi_{1}^{\prime}, \xi_{2}^{\prime}\right)-\left|f\left(\xi_{1}^{\prime}, \xi_{2}^{\prime}\right)\right|^{2}\right]+\lambda\right) \mathrm{e}^{-i\left(c_{1} \xi_{\xi_{1}^{\prime}}+c_{2} m \xi_{2}^{\prime}\right)} \\
\times \sum_{n=-M_{1}}^{M_{1}} \sum_{m=-M_{2}}^{M_{2}} I_{n m} \mathrm{e}^{i\left(c_{1} \xi_{1}^{\prime}+c_{2} m \xi_{2}^{\prime}\right)} \mathrm{d} \xi_{1}^{\prime} \mathrm{d} \xi_{2}^{\prime}, \\
n=\overline{-M_{1}, M_{1}}, m=\overline{-M_{2}, M_{2}}
\end{gathered}
$$

from the necessary condition of minimum of (32). The respective Euler's equation (6), for the functional (32) is

$$
\begin{aligned}
f\left(\xi_{1}, \xi_{2}\right)= & \int_{-1}^{1} \int_{-1}^{1}\left(2\left[F^{2}\left(\xi_{1}^{\prime}, \xi_{2}^{\prime}\right)-\left|f\left(\xi_{1}^{\prime}, \xi_{2}^{\prime}\right)\right|^{2}\right]+\lambda\right) \\
& \times K\left(\xi_{1}, \xi_{2}, \xi_{1}^{\prime}, \xi_{2}^{\prime}, c_{1}, c_{2}\right) f\left(\xi_{1}^{\prime}, \xi_{2}^{\prime}\right) \mathrm{d} \xi_{1}^{\prime} \mathrm{d} \xi_{2}^{\prime},
\end{aligned}
$$

where the kernel $K$ is a real and it is calculated by

$$
\begin{aligned}
K\left(\xi_{1}, \xi_{2}, \xi_{1}^{\prime}, \xi_{2}^{\prime}, c_{1}, c_{2}\right)= & \sum_{n=-M_{1}}^{M_{1}} \sum_{m=-M_{2}}^{M_{2}} \mathrm{e}^{i\left[c_{1} n\left(\xi_{1}^{\prime}-\xi_{1}^{\prime}\right)+c_{2} m\left(\xi_{2}-\xi_{2}^{\prime}\right)\right]} \\
& =\frac{\sin N_{1} \frac{c_{1}}{2}\left(\xi_{1}-\xi_{1}^{\prime}\right)}{\sin \frac{c_{1}}{2}\left(\xi_{1}-\xi_{1}^{\prime}\right)} \cdot \frac{\sin N_{2} \frac{c_{2}}{2}\left(\xi_{2}-\xi_{2}^{\prime}\right)}{\sin \frac{c_{2}}{2}\left(\xi_{2}-\xi_{2}^{\prime}\right)} .
\end{aligned}
$$

The Lagrange multiplier $\lambda$ is determined by a coordinate descent method

$$
\lambda_{k+1}=\lambda_{k}-\gamma_{k} \frac{\partial L\left(I^{(k)}, \lambda_{k}\right)}{\partial \lambda},
$$

while the value $\gamma_{k}$ at each step will be calculated using the relation:

$$
\gamma_{k}=\frac{L\left(I^{(k)}, \lambda_{k}\right)}{\left\|\frac{\partial L\left(I^{(k)}, \lambda_{k}\right)}{\partial \lambda}\right\|^{2}} .
$$

Consequently, the iterative process (8)-(9) looks like

$$
\begin{aligned}
I_{n m}^{(k+1)}= & \frac{1}{\alpha} \int_{-1}^{1} \int_{-1}^{1}\left(2\left[P\left(\xi_{1}^{\prime}, \xi_{2}^{\prime}\right)-\left|f^{(k)}\left(\xi_{1}^{\prime}, \xi_{2}^{\prime}\right)\right|^{2}\right]+\lambda_{k}\right) \mathrm{e}^{-i\left(c_{1} n \xi_{1}^{\prime}+c_{2} m \xi_{2}^{\prime}\right)} \\
& \times \sum_{n=-M_{1}}^{M_{1}} \sum_{m=-M_{2}}^{M_{2}} I_{n m}^{(k)} \mathrm{e}^{i\left(c_{1} n \xi_{1}^{\prime}+c_{2} m \xi_{2}^{\prime}\right)} \mathrm{d} \xi_{1}^{\prime} \mathrm{d} \xi_{2}^{\prime},
\end{aligned}
$$




$$
\lambda_{k+1}=\lambda_{k}+\frac{L\left(I_{n m}^{(k)}, \lambda_{k}\right)}{\left\|\frac{\partial L\left(I_{n m}^{(k)}, \lambda_{k}\right)}{\partial \lambda}\right\|^{2}}\left(\left\|f^{(k)}\left(\xi_{1}, \xi_{2}\right)\right\|^{2}-\left\|P\left(\xi_{1}, \xi_{2}\right)\right\|\right), \quad k=0,1, \cdots
$$

Numerical experiments of synthesis by the algorithm (38), (39) for different values of parameters $c_{1}$ and $c_{2}$ and different power RPs $F^{2}\left(\xi_{1}, \xi_{2}\right)$ show that, firstly, there are non-zero solutions of the nonlinear Equation (34) and, secondly, solutions that differ from zero with increasing parameters $c_{1}$ and $c_{2}$ become more effective not only in understanding the values of the functional (32), but also in the form. In particular, for a plane equidistant array, which consists of $121(11 \times 11)$ radiators and a given power $\mathrm{RP} F^{2}\left(\xi_{1}, \xi_{2}\right)=1$, the values of the functional (32), which it acquires at the optimal solution for different values of the parameters $c_{1}$ and $c_{2}$, are given in Table 1 , and the optimal solutions (synthesized RPs) for some set of the parameters $c_{1}$ and $c_{2}$ are shown in Figures 3-5. One can see that such prescribed power RP $F^{2}$ can be not approximated with sufficient accuracy at the given number of radiators don't hurt the frequency (parameter $c$ and wave number $k$ respectively). In order to achieve better approximation for such RP, there is necessary to increase the number of radiators (and size of array respectively) in a great extent.

Even more effective solutions exist for RPs that do not have central symmetry. In Figure 6, the results of synthesis are presented for given two-lobe power RP

Table 1. Values of functional at the different $c_{1}, c_{2}$.

\begin{tabular}{cc}
\hline Parameters $\quad c_{1}=c_{2}$ & Functional $(32) L(I, \lambda)$ \\
\hline 0.57 & 0.759321 \\
0.75 & 0.603479 \\
1.0 & 0.559591 \\
1.2 & 0.518555 \\
1.4 & 0.491708 \\
\hline
\end{tabular}

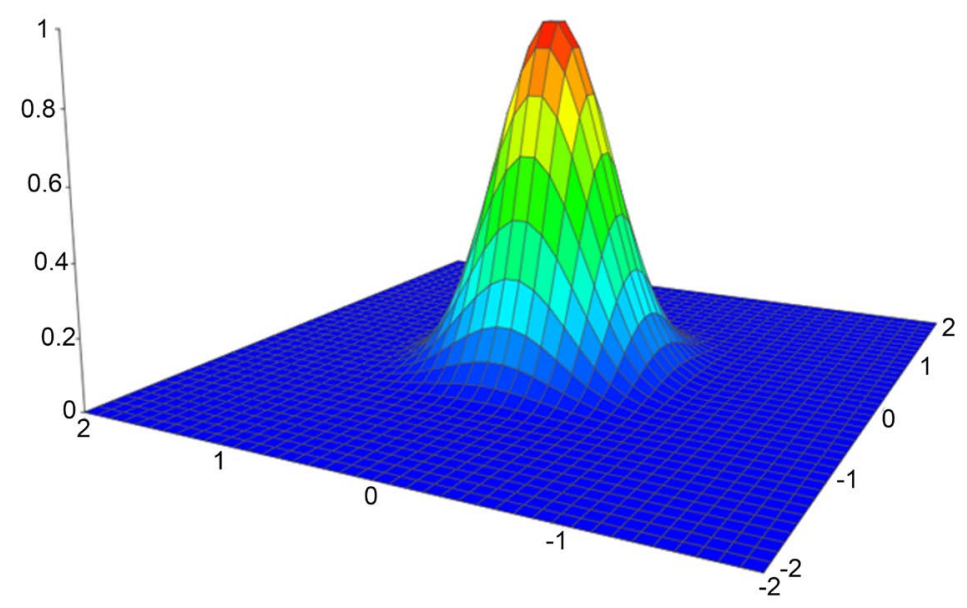

Figure 3. Synthesized power RP at $c_{1}=0.57, c_{2}=0.57, F^{2}\left(\xi_{1}, \xi_{2}\right)=1$. 


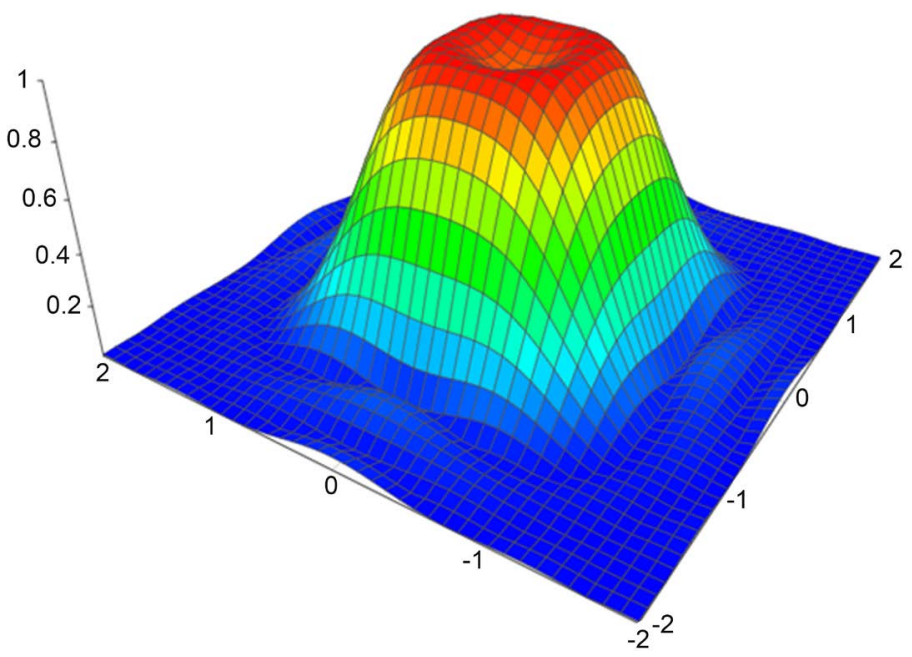

Figure 4. Synthesized power RP at $c_{1}=1.0, c_{2}=1.0, F^{2}\left(\xi_{1}, \xi_{2}\right)=1$.

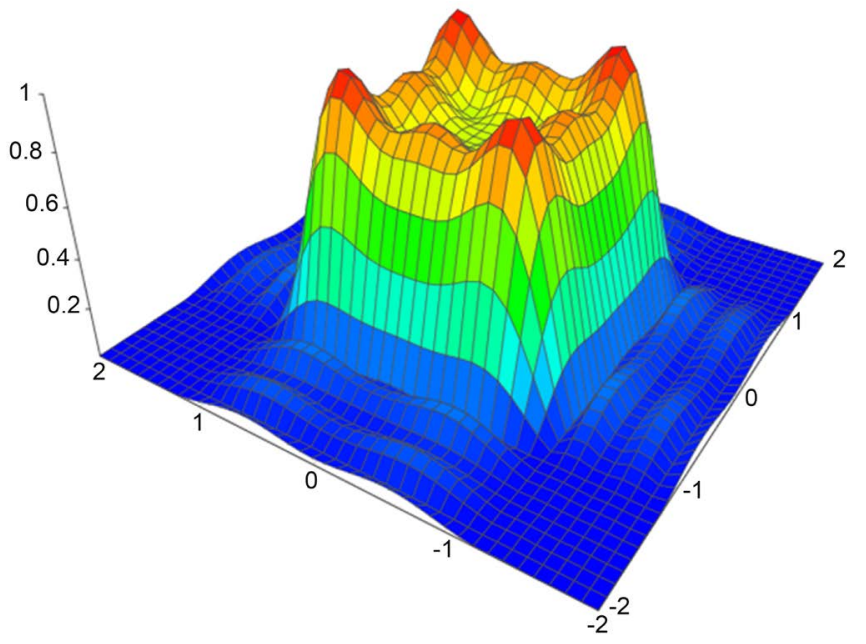

Figure 5. Synthesized power RP at $c_{1}=1.4, c_{2}=1.4, F^{2}\left(\xi_{1}, \xi_{2}\right)=1$.

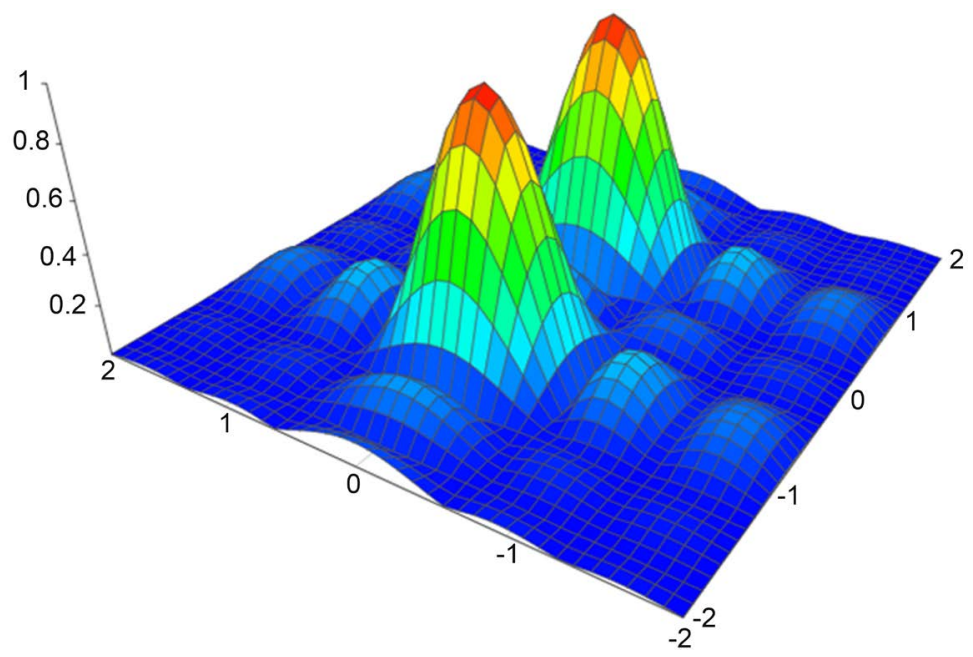

Figure 6. Synthesized power RP at $c_{1}=0.85, \quad c_{2}=1.2, \quad F^{2}\left(\xi_{1}, \xi_{2}\right)=\cos ^{2}\left(\frac{\pi \xi_{1}}{2}\right)\left|\sin \pi \xi_{2}\right|^{2}$. 
$F^{2}\left(\xi_{1}, \xi_{2}\right)=\cos ^{2}\left(\frac{\pi \xi_{1}}{2}\right)\left|\sin \pi \xi_{2}\right|^{2}$ and parameters $c_{1}=0.85, c_{2}=1.2$. One can see that the synthesized RP good approximates the given one not only in the sense of mean square approximation (values of functional (32) $L(I, \lambda)=0.070109$ ), but also with respect of the form. The difference of given and synthesized RPs in the main lobe does not exceed $0.052(-25.68 \mathrm{~dB})$, and the level of side lobes does not exceed $0.072(-22.85 \mathrm{~dB})$. For the constant RP $F^{2}$ these values are much worse: $0.12(-18.41 \mathrm{~dB})$ and $0.17(-15.39 \mathrm{~dB})$ respectively.

\section{Conclusions}

A generalized statement of the synthesis problem according to a given power RP was considered. Taking into account of additional conditions on the norm of the synthesized power RP ensures the absence of zero solutions. This can not be provided if the usual statement of the synthesis problem according to given power RP is used. The synthesis problem was examined for two types of antennas.

In the case of simpler operator calculation of RP by the currents in linear antenna, the analytical-numerical procedure for investigation of non-uniqueness of solutions to respective non-linear integral equation of Hammerstein's type was elaborated and the numerical results related to the qualitative and quantitative properties of obtained solutions were presented. The modified Newton's method was applied successfully to obtain the solutions with the different properties of phase of the synthesized power RP.

In the case of plane equidistant array, for which the operator for calculations of RP by the currents in array radiators is more complicate, the numerical procedures were applied for solving the synthesis problems for the specific given RPs. The approach proposed foresees the application for other types of antenna systems, for which the RP is calculated by the currents (fields) in antenna elements by known linear operator.

\section{Conflicts of Interest}

The authors declare no conflicts of interest regarding the publication of this paper.

\section{References}

[1] Liu, Y.H., Huang, X., Xu, K.D., Song, Z.Y., Yang, S.W. and Liu, Q.H. (2017) Pattern Synthesis of Unequally Spaced Linear Arrays including Mutual Coupling Using Iterative FFT via Virtual Active Element Pattern Expansion. IEEE Transactions on Antennas and Propagation, 65, 3950-3958. https://doi.org/10.1109/TAP.2017.2708081

[2] Rivas, A., Rodriguez, J.A., Ares, F. and Moreno, E. (2001) Planar Arrayas with Square Lattices and Circular Boundaries: Sum Patterns from Distributions with Uniform, Amplitude or Very Low Dynamic-Range Ratio. IEEE Antennas and Propagation Magazine, 43, 90-93. https://doi.org/10.1109/74.979369 
[3] Bhattacharyya, A.K. (2007) Active Element Pattern Symmetry for Asymmetrical Element Arrays. IEEE Antennas and Wireless Propagation Letters, 6, 275-278. https://doi.org/10.1109/LAWP.2007.898549

[4] Morabito, A.E., Isernia, T. and D’Urso, M. (2010) Synthesis of Difference Patterns via Uniform Amplitude Sparse Arrays. Electronics Letters, 46, 554-556. https://doi.org/10.1049/el.2010.3591

[5] Sobhani, H., Zakeri-Khatir, H. and Firouzjaei, A.S. (2018) Controlling Amplitude and Radiation Pattern of Emitted Terahertz by Group Velocity Mismatch. IEEE Transactions on Antennas and Propagation, 66, 5268-5273. https://doi.org/10.1109/TAP.2018.2854304

[6] Bulatsyk, O.O., Katsenelenbaum, B.Z., Topolyuk, Y.P. and Voitovich, N.N. (2010) Phase Optimization Problems. Wiley-VCH, Weinheim.

https://doi.org/10.1002/9783527629824

[7] Choni, Y.I. (1968) On Antenna System Synthesis According to Given Amplitude Radiation Pattern. Izvestiya Vuzov-Radioelectronika, 11, 1325-1327. (In Russian)

[8] Voitovich, N.N. (1972) Antenna Synthesis according to Amplitude Radiation Pattern (V. V. Semenov's Method). Radio Engineering and Electronics, 17, 2491-2497.

[9] Voitovich, N.N. and Savenko, P.A. (1975) Branching of Solutions of the Antenna Synthesis Problem Based on a Specified Amplitude Radiation Pattern. Radio Engineering and Electronics, 20, 1-8.

[10] Savenko, P.A. (1979) Synthesis of Linear Antenna Arrays with the Prescribed Amplitude Pattern. Radio Engineering and Electronics, 22, 1045-1049.

[11] Andriychuk, M.I., Voitovich, N.N., Savenko, P.A. and Tkachuk, V.P. (1993) Synthesis of Antennas According to Amplitude Directivity Pattern. Naukova Dumka, Kiev. (In Russian)

[12] Podlevskyi, B.M. (2009) Numerical Algorithms of Finding the Branching Lines and Bifurcation Points of Solutions of Nonlinear Integral Equation Arising in the Theory of Antennas Synthesis. International Seminar/ Workshop on Direct and Inverse Problems of Electromagnetic and Acoustic Wave Theory, Lviv, 21-24 September 2009, 197-203. https://doi.org/10.1109/DIPED.2009.5306942

[13] Podlevs'kyi, B.M. (2010) On One Approach to Finding the Branching Lines and Bifurcation Points of Solutions of Nonlinear Integral Equations Whose Kernels Depend Analytically on Two Spectral Parameters. Journal of Mathematical Sciences, 171, 433-452. https://doi.org/10.1007/s10958-010-0148-y

[14] Podlevskyi, B.M. (2010) On Some Nonlinear Two-Parameter Spectral Problems of Mathematical Physics. Mathematical Modelling, 22, 131-145. (In Russian)

[15] Podlevskyi, B.M. (2012) Numerical Algorithms of Finding the Branching Lines and Bifurcation Points of Solutions for One Class of Nonlinear Integral Equations. In: Awrejcewicz, J. and Hagedorn, P., Eds., Nonlinearity, Bifurcation and Chaos. Theory and Applications, InTech Publishing, Novi Sad, 281-312.

[16] Podlevskyi, B.M., Khlobystov, V.V. and Yaroshko, O.S. (2017) Multiparameter Eigenvalue Problems: Methods and Algorithms. Lambert Acad. Publish.

[17] Savenko, P.O. (2000) On Structure of Solution to Problem of Synthesis of Linear Antenna by a Given Radiation Pattern. Radiophysics and Radioastronomy, 5, 405-415. (In Russian)

[18] Savenko, P.O. (2002) Nonlinear Problems of Radiating Systems. IAPMM NASU, Lviv. (In Ukrainian)

[19] Savenko, P.O. (2014) Nonlinear Synthesis Problems of Radiating Systems with 
Plane Aperture. IAPMM NASU, Lviv. (In Ukrainian)

[20] Podlevskyi, B.M. and Mohytych, H.A. (2015) Numerical Algorithm for Finding Branching Points for Solutions of a Class of Nonlinear Integral Equations. International Scientific Mykhailo Kravchuk Conference, Vol. 2, Kyiv, May 13-15 2015, 149-153.

[21] Podlevskyi, B.M. and Koval, T.V. (2016) Numerical Algorithm for Finding Branching Points for Solutions of a Class of Nonlinear Integral Equations. International Scientific Mykhailo Kravchuk Conference, Vol. 2, Kyiv, May 19-20 2016, 151-154.

[22] Andriychuk, M.I. and Voitovich, N.N. (2013) Antenna Synthesis According to Power Radiation Pattern with Condition of Norm Equality. Seminar/ Workshop on Direct and Inverse Problems of Electromagnetic and Acoustic Wave Theory, Lviv, 137-140.

[23] Voitovich, N.N., Topolyuk, Yu.P. and Reshnyak, O.O. (2000) Approximation of Compactly Supported Functions with Free Phase by Functions with Bounded Spectrum. Fields Institute Communications, 25, 531-541.

[24] Voitovich, N.N. (2003) Antenna Synthesis by Amplitude Radiation Pattern and Modified Phase Problem. In: Katsenelenbaum, B.Z., Ed., Electromagnetic FieldsRestrictions and Approximation, WILEY-VCH, Weinheim, 195-233.

[25] Bulatsyk, O.O. (2013) Complex Polynomials Representation of Solutions to the Antenna Synthesis Problem by the Power Pattern. Seminar/Workshop on Direct and Inverse Problems of Electromagnetic and Acoustic Wave Theory, Lviv, 166-170.

[26] Andriychuk, M., Bulatsyk, O. and Voitovich, N. (2014) Polynomial Approach to Linear Antenna Array Synthesis Problem According to Power Radiation Pattern with Restriction to Its Norm. 15th International Conference on Mathematical Methods in Electromagnetic Theory, Dnipropetrovsk, 36-38. https://doi.org/10.1109/MMET.2014.6928739

[27] Andriychuk, M.I., Bulatsyk, O.O. and Voitovich, N.N. (2014) Comparing Different Approaches to Linear Antenna Synthesis Problems According to Power Radiation Pattern. Seminar/Workshop on Direct and Inverse Problems of Electromagnetic and Acoustic Wave Theory, Tbilisi, 15-18. https://doi.org/10.1109/DIPED.2014.6958307

[28] Andriychuk, M. and Voytovych, M. (2014) Modified Newton Method for Antenna Power Synthesis Problem with Fixed Norm of the Pattern. Journal of Computational and Applied Mathematics, 2, 3-10.

[29] Andriychuk, M.I., Bulatsyk, O.O. and Voytovych, M.M. (2014) Applying the Concept of Generating Polynomials to the Antenna Synthesis Problem by Power Criterion. Mathematical Modeling and Computing, 1, 121-134. 